\title{
Multiplicities and Reduction Numbers
}

\author{
WOLMER V. VASCONCELOS \\ Department of Mathematics, Rutgers University, 110 Frelinghuysen Road, Piscataway, \\ NJ 08854-8019, U.S.A. e-mail: vasconce@math.rutgers.edu
}

(Received: 11 February 2002; accepted in final form: 16 September 2002)

\begin{abstract}
Let $(R, \mathfrak{m})$ be a Cohen-Macaulay local ring and let $I$ be an ideal. There are at least five algebras built on $I$ whose multiplicity data affect the reduction number $r(I)$ of the ideal. We introduce techniques from the Rees algebra theory of modules to produce estimates for $r(I)$, for classes of ideals of dimension one and two. Previous cases of such estimates were derived for ideals of dimension zero.
\end{abstract}

Mathematics Subject Classifications (2000). Primary: 13H15; secondary: 13D40, $13 \mathrm{H} 10$.

Key words. Cohen-Macaulay ring, conormal module, Hilbert function, multiplicity, reduction number, Rees algebra.

\section{Introduction}

Let $R$ be a commutative Noetherian ring, and let $I$ be one of its ideals. A reduction of $I$ is an ideal $J \subset I$ such that $I^{n+1}=J I^{n}$. The least such integer $n$ is the reduction number of $I$ relative to $J$, and is denoted by $r_{J}(I)$. Reductions play a role in the theory of finite morphisms of the blowup $\operatorname{Blow}_{V(I)}(\operatorname{Spec}(R)$, with the reduction number being a control element. In case $R$ is a local ring (of infinite residue field), minimal reductions are particularly valuable because they help control the cohomology of the blowup. We are interested in the minimum value of $r_{J}(I)$, which we denote by $r(I)$, from among all possible minimal reductions.

Reduction numbers are derivative invariants of $I$, often measuring the interplay amongst the 'primary' invariants of the ideal. It can achieve very low values, as in the case when $R$ is a Cohen-Macaulay local ring and the Rees algebra of $I$ is also Cohen-Macaulay: according to Johnston and Katz (1995), $r(I)<\operatorname{dim} R$. Several of these notions can be defined on graded algebras and $R$-modules, and here we want to exploit this even while we focus on ideals.

In this paper we shall seek to extend to ideals of positive dimension the quadratic relationships between the reduction number $r(I)$ and various multiplicities that were developed in Doering et al. (1998) and Vasconcelos (1998a) for ideals of dimension zero. Figuratively, for an ideal, module or algebra $A$ (graded in the appropriate case), where the notions of reduction number $r(A)$, multiplicity $d(A)$ and dimension $d$ have been defined, there are usually no direct relationships between these quantities. More correctly, the relationship between $r(A)$ and $d(A)$ is mediated through 
various agents such as dimensions, Castelnuovo-Mumford regularity, Hilbert function, and numerical information in the syzygies associated to one of the various algebras built on $I$ :

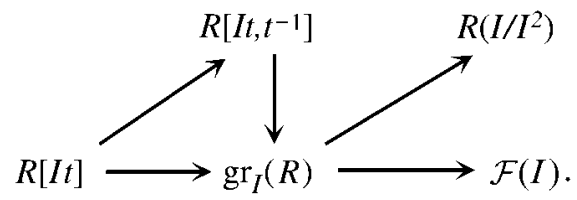

We want to capture these possibilities by inequalities of the form

$$
r(A) \leqslant f(d(A), \operatorname{dim} A),
$$

where $d(A)$ is some 'degree' of $A(\operatorname{deg}(A)$, arith-deg $(A)$ or even some extended degree $\operatorname{Deg}(A)$ Doering et al. (1998), and $f$ is a polynomial of low degree. For example, if $I$ is an $\mathrm{m}$-primary ideal and the residue field of $R$ has characteristic zero, then

$$
r(I) \leqslant \operatorname{arith}-\operatorname{deg}\left(\operatorname{gr}_{I}(R)\right),
$$

where $\operatorname{gr}_{I}(R)$ is the associated graded ring of $I$.

There are other bounds mediated by other agents. According to Vasconcelos (1998a), for a Cohen-Macaulay local ring $(R, \mathfrak{m})$ of Krull dimension $d$, an $\mathfrak{m}$-primary ideal $I$ of multiplicity $e(I)$ satisfies

$$
r(I) \leqslant(d-h) e(I)-2(d-h)+1,
$$

where $h$ is some integer $h<d$ such that depth $\operatorname{gr}_{I}(R) \geqslant h$. The method of Vasconcelos (1998) is dependent on the Cohen-Macaulayness of $R$ and on $I$ being an m-primary ideal. To extend to more general rings required in Rossi et al. (2001) replacing $e(I)$ by the length $\lambda(R / J)$, where $J$ is a minimal reduction of $I$.

The reduction number of an ideal is actually a property of its fiber cone, $\mathcal{F}(I)=\operatorname{gr}_{I}(R) \otimes(R / \mathfrak{m})$, and therefore a major issue is how $I$ affects the properties of $\mathcal{F}(I)$. For example, if the residue field of $R$ has characteristic zero,

$$
\mathrm{r}(I)<\operatorname{arith}-\operatorname{deg}(\mathcal{F}(I)) .
$$

To refine these bounds, in a manner that is responsive to other properties of $I$, is a challenge. It is a difficult task to track the properties of $I$ in its passage to $\mathcal{F}(I)$. A practical approach to this problem begins by attempting to bound the Hilbert function of the fiber cone of $I, v\left(I^{n}\right)$ for all $n$, by a polynomial $f(n)$ of degree $s-1, f(n)$ depending on the various extended multiplicities of $I$. Solving for the least solution of

$$
f(n)<\left(\begin{array}{c}
n+s \\
s
\end{array}\right)
$$


which may require a convenient form for $f(n)$, and using a result of Eakin and Sathaye (1976), gives $n>\mathrm{r}(I)$. This provides for a reduction $L$ generated by $s$ elements and an attached bound for $\mathrm{r}_{L}(I)$. In case $I$ is m-primary, $s=\ell(I)$, and $L$ is a minimal reduction. If however $\operatorname{dim} R / I \geqslant 1$, this approach only provides for a reduction $L$ with fewer generators. It is helpful however to find a minimal reduction $J$ of $L$ in order to apply $\mathrm{r}_{J}(I) \leqslant \mathrm{r}_{L}(I)+\mathrm{r}_{J}(L)$.

We will take two paths to these estimations. First, by extending to ideals of positive dimension the methods that have already been used for ideals of finite co-length. This required the development of techniques to bound the number of generators of powers of ideals - essentially a Hilbert function - by filtering them (whenever possible) in such a way that the factors are Cohen-Macaulay. This approach led to a number of results in Sections 2 and 3 that benefit from the more amenable relationships between multiplicities and number of generators of Cohen-Macaulay modules. One unpleasant technical aspect of these bounds, for ideals which are not of finite co-length, is the presence of factorial terms, a feature that could be bypassed in the special case of dimension zero. Nevertheless an opening is provided by the consideration of the Rees algebra of the conormal module. It is a small gap but sufficiently wide to lead to several other cases of ideals of low dimension.

Our main results are treated in Section 4, whose aim is to derive analogs of (1) and (2) for ideals of dimension 1 and 2. Our two main results are the following. In both, $R$ is a Cohen-Macaulay local ring of dimension $d$, with an infinite residue field. For an ideal $I$, we set $\mathcal{G}=\operatorname{gr}_{I}(R)$ for its associated graded ring.

- Theorem 4.6. If $I$ is a Cohen-Macaulay ideal of dimension 1 that is generically a complete intersection, then

$$
\mathrm{r}(I)<\operatorname{deg}(\mathcal{G}) .
$$

- Theorem 4.10. If $I$ is a perfect Gorenstein ideal of dimension 2 and $R / I$ is normal, then

$$
\mathrm{r}(I) \leqslant(d-1) \operatorname{deg}(\mathcal{G})-4 d+5 .
$$

Actually, both estimates come close to bounding the reduction numbers $r_{J}(I)$ for an arbitrary reduction $J$ of $I$, the so-called big reduction number $\operatorname{br}(I)$ of $I$.

\section{General Techniques}

In this section we convert quickly the techniques used in Doering et al. (1998) to estimate the number of generators of the powers $I^{n}$ of certain ideals.

Basic results, general terminology and notation will be that of Bruns and Herzog (1993), except when we use $\lambda(\cdot)$ for the length function on modules with a composition series. As usual, the minimum number of generators function is denoted by $v(\cdot)$.

We begin with a brief review of the notions of multiplicity that will be used, and refer to Vasconcelos (1998b), Chapter 9 for a detailed discussion. Let $(R, \mathfrak{m})$ 
be a Noetherian local ring (or a graded ring with $\mathfrak{m}$ denoting the maximal homogeneous ideal), and let $\mathcal{M}(R)$ be the category of finitely generated modules (with suitable morphisms). A degree is a numerical function

$$
\operatorname{deg}: \mathcal{M}(R) \mapsto \mathbb{N},
$$

with appropriate properties. The outstanding example is the ordinary multiplicity,

$$
\operatorname{deg}(M)=d ! \cdot \lim _{n \rightarrow \infty} \frac{\lambda\left(M / \mathfrak{m}^{n} M\right)}{n^{d}},
$$

where $d=\operatorname{dim} M$. If $I$ is an $m$-primary ideal and $\mathcal{G}$ denotes its associated graded ring,

$$
\mathcal{G}=\operatorname{gr}_{I}(R)=\sum_{n \geqslant 0} I^{n} / I^{n+1},
$$

we set $e(I)=\operatorname{deg}(\mathcal{G})$, and refer to it as the multiplicity of $I$. The analytic spread of $I$ is the dimension of the special fiber $\ell(I)=\operatorname{dim} \mathcal{F}(I)$.

This terminology extends to a graded algebra $A$, finitely generated over a local ring $(R, \mathfrak{m}): \ell(A)=\operatorname{dim} A / \mathfrak{m} A$. However, we use $\operatorname{deg}(A)$ to denote the multiplicity of $A$ relative to its (unique) maximal homogeneous ideal.

If one wants to capture the contributions of each primary component of the module, adding them all gives rise to the arithmetic degree of $M$, arith-deg $(M)$. Assuming $R$ is Gorenstein (for simplicity) it assembles itself into

$$
\operatorname{arith}-\operatorname{deg}(M)=\sum_{i=0}^{n} \operatorname{deg}\left(\operatorname{Ext}_{R}^{i}\left(\operatorname{Ext}_{R}^{i}(M, R), R\right)\right) \text {. }
$$

An additional refinement was made by Doering et al. (1998) with the introduction of functions $\operatorname{Deg}(\cdot): \mathcal{M}(S) \mapsto \mathbb{N}$, satisfying

(i) If $L=\Gamma_{\mathfrak{m}}(M)$ is the submodule of elements of $M$ which are annihilated by a power of the maximal ideal and $\bar{M}=M / L$, then

$$
\operatorname{Deg}(M)=\operatorname{Deg}(\bar{M})+\ell(L) .
$$

(ii) (Bertini's rule) If $M$ has positive depth and $h \in R$ is a generic hyperplane section on $M$, then

$$
\operatorname{Deg}(M) \geqslant \operatorname{Deg}(M / h M)
$$

(iii) (The calibration rule) If $M$ is a Cohen-Macaulay module, then

$$
\operatorname{Deg}(M)=\operatorname{deg}(M) .
$$

There are several such functions if $\operatorname{dim} R \geqslant 2$, and any of them will be denoted $\operatorname{Deg}(\cdot)(\mathrm{read}$ : big $\operatorname{deg}(\cdot))$. 


\subsection{CODIMENSION MINUS ONE TRICK}

Let $(R, \mathfrak{m})$ be a Cohen-Macaulay local ring of dimension $d>0$, with an infinite residue field, and let $I$ be an ideal of height $g<d$; set $\ell=\ell(I)$ for the analytic spread of $I$. Let $J_{0}=\left(a_{1}, \ldots, a_{h}\right)$ be part of a minimal reduction of $I$, with the $a_{i}$ 's forming a regular sequence. Now from the embedding $I^{n} / J_{0}^{n} \hookrightarrow R / J_{0}^{n}$, one has

$$
v\left(I^{n}\right) \leqslant v\left(J_{0}^{n}\right)+v\left(I^{n} / J_{0}^{n}\right) .
$$

We need to bound the right-hand side by a polynomial $f(n)$ of degree at most $\ell-1$ in order to apply the comments above. The first term is simply

$$
v\left(J_{0}^{n}\right)=\left(\begin{array}{c}
n+h-1 \\
h-1
\end{array}\right),
$$

so it presents no difficulty since $h \leqslant g \leqslant \ell \leqslant d$. The other term arises from an ideal of the Cohen-Macaulay $S=R / J_{0}^{n}$, whose properties are traceable to those of $R$ and ordinary multiplicities of $R / I$. The heart of the matter is to estimate the number of generators of the ideal $I^{n} / J_{0}^{n}$, using some multiplicity of $S$ but still bounded by a polynomial of degree $\ell-1$.

Two variants can be introduced: (i) Since one can use other kinds of multiplicities, some of which may work well for non Cohen-Macaulay rings, we do not have to choose $J_{0}$ to be a complete intersection. (ii) To achieve a minimal reduction $J$ with the desirable reduction number, one may first use the technique to achieve an intermediate reduction $L$, as indicated above.

Let us indicate how this works in practice by extending a result of Doering et al. (1998) to a much wider class of filtrations.

PROPOSITION 2.1. Let $(R, \mathfrak{m})$ be a Cohen-Macaulay local ring of dimension $d \geqslant 1$ and infinite residue field. Let $\mathfrak{\wp}=\left\{I_{n}, n \geqslant 1\right\}$ be a multiplicative filtration of Cohen-Macaulay ideals of dimension $d-g<d$. If $I=I_{1}$ is an equimultiple ideal then for all $n$

$$
v\left(I_{n}\right) \leqslant g ! \operatorname{deg}(R / \bar{I}) \operatorname{deg}(R)\left(\begin{array}{c}
n+g-2 \\
g-1
\end{array}\right)+\left(\begin{array}{c}
n+g-2 \\
g-2
\end{array}\right) .
$$

Proof. We pick $J_{0}$ as above: $J_{0}=\left(a_{1}, \ldots, a_{g-1}\right)$ is an ideal of height $g-1$ with the $a_{i}$ 's being part of a minimal reduction of $I$. This already accounts for the summand

$$
v\left(J_{0}^{n}\right)=\left(\begin{array}{c}
n+g-2 \\
g-2
\end{array}\right) .
$$

By the other assumption, $I_{n} / J_{0}^{n}$ is a Cohen-Macaulay ideal of height 1 of the Cohen-Macaulay ring $R / J_{0}^{n}$. Thus $I_{n} / J_{0}^{n}$ is a Cohen-Macaulay module and therefore

$$
v\left(I_{n} / J_{0}^{n}\right) \leqslant \operatorname{deg}\left(R / J_{0}^{n}\right)=\operatorname{deg}\left(R / J_{0}\right)\left(\begin{array}{c}
n+g-2 \\
g-1
\end{array}\right) .
$$


Let $J=\left(J_{0}, x\right)$ be a minimal reduction of $I$. Since $x$ is regular module $J_{0}$, we have $\operatorname{deg}\left(R / J_{0}\right) \leqslant \operatorname{deg}(R / J)$.

What we need will be provided in a estimate of multiplicities based on a wellknown theorem (Lech, 1960):

THEOREM 2.2. Let $(R, \mathfrak{m})$ be a Noetherian local ring of dimension $d$ and let $I$ be an m-primary ideal. Then

$$
e(I) \leqslant d ! \cdot \operatorname{deg}(R) \cdot \lambda(R / \bar{I}) .
$$

We formulate it replacing $I$ by its integral closure $\bar{I}$, which can only sharpen the inequality.

PROPOSITION 2.3. Let $R$ be a Cohen-Macaulay local ring of infinite residue field and let $I$ be an equimultiple ideal of codimension $g$. If $J$ is a minimal reduction of I then

$$
\operatorname{deg}(R / J) \leqslant g ! \operatorname{deg}(R / \bar{I}) \operatorname{deg}(R) .
$$

Proof. Consider how the associativity formula for multiplicities relates to $R / J$ :

$$
\operatorname{deg}(R / J)=\sum_{\mathfrak{p}} \lambda\left(R_{\mathfrak{p}} / J_{\mathfrak{p}}\right) \operatorname{deg}(R / \mathfrak{p}),
$$

where $\mathfrak{p}$ runs over the minimal primes of $J$; since $I$ is equimultiple, they are the same as the minimal primes of $I$.

We note that $\lambda\left(R_{\mathfrak{p}} / J_{\mathfrak{p}}\right)$ is the Samuel multiplicity of the ideal $I_{\mathfrak{p}}$. By Theorem 2.2,

$$
e\left(I_{\mathfrak{p}}\right) \leqslant g ! \lambda\left(R_{\mathfrak{p}} / \bar{I}_{\mathfrak{p}}\right) \operatorname{deg}\left(R_{\mathfrak{p}}\right) .
$$

Noting that $\operatorname{deg}\left(R_{\mathfrak{p}}\right) \leqslant \operatorname{deg}(R)$, we obtain

$$
\begin{aligned}
\operatorname{deg}(R / J) & =\sum_{\mathfrak{p}} e\left(I_{\mathfrak{p}}\right) \operatorname{deg}(R / \mathfrak{p}) \\
& \leqslant g ! \sum_{\mathfrak{p}} \lambda\left(R_{\mathfrak{p}} / \bar{I}_{\mathfrak{p}}\right) \operatorname{deg}(R) \operatorname{deg}(R / \mathfrak{p}) \\
& =g !\left(\sum_{\mathfrak{p}} \lambda\left(R_{\mathfrak{p}} / \bar{I}_{\mathfrak{p}}\right) \operatorname{deg}(R / \mathfrak{p})\right) \cdot \operatorname{deg}(R) \\
& =g ! \operatorname{deg}(R / \bar{I}) \operatorname{deg}(R),
\end{aligned}
$$

giving the asserted bound.

\subsection{MULTIPLICITY OF THE SPECIAL FIBER}

We give an application of the method to derive estimates for the multiplicity of the special fiber of $\mathfrak{m}$-primary ideals. Let $(R, \mathfrak{m})$ be a Cohen-Macaulay local ring of dimension $d$ and let $I$ be an $\mathrm{m}$-primary ideal. The surjection of $d$-dimensional rings

$$
0 \rightarrow H \longrightarrow \operatorname{gr}_{I}(R) \longrightarrow \mathcal{F}(I) \rightarrow 0
$$


gives the inequality $\operatorname{deg}\left(\operatorname{gr}_{I}(R)\right)=e(I) \geqslant \operatorname{deg}(\mathcal{F}(I))$. The following observation permits the derivation of another kind of bound, one that involves the next coefficient, $e_{1}(I)$, of the Hilbert polynomial of $I$. Let $J=\left(a_{1}, \ldots, a_{d}\right)$ be a minimal reduction of $I$, and consider the exact sequence of modules of finite length

$$
0 \rightarrow I^{n} / J^{n} \longrightarrow R / J^{n} \longrightarrow R / I^{n} \rightarrow 0 .
$$

Taking lengths, we have

$$
\lambda\left(I^{n} / J^{n}\right)=\lambda\left(R / J^{n}\right)-\lambda\left(R / I^{n}\right)=e(I)\left(\begin{array}{c}
d+n-1 \\
d
\end{array}\right)-\lambda\left(R / I^{n}\right) .
$$

For $n \gg 0$, replacing $\lambda\left(R / I^{n}\right)$ by the Hilbert polynomial of $I$, we obtain

$$
\lambda\left(I^{n} / J^{n}\right)=e_{1}(I)\left(\begin{array}{c}
n+d-2 \\
d-1
\end{array}\right)+\text { lower terms, }
$$

from the cancelling of the term $e(I)\left(\begin{array}{c}n+d-1 \\ d\end{array}\right)$ in $\lambda\left(R / I^{n}\right)$.

PROPOSITION 2.4. Let $(R, \mathfrak{m})$ be a Cohen-Macaulay local ring and let I be an m-primary ideal. Then

$$
\operatorname{deg}(\mathcal{F}(I)) \leqslant \inf \left\{e_{0}(I), e_{1}(I)+1\right\} .
$$

Proof. From the estimate for the number of generators of $I$

$$
v\left(I^{n}\right) \leqslant v\left(J^{n}\right)+v\left(I^{n} / J^{n}\right) \leqslant v\left(J^{n}\right)+\lambda\left(I^{n} / J^{n}\right),
$$

for $n \gg 0$ we obtain

$$
v\left(I^{n}\right) \leqslant\left(\begin{array}{c}
n+d-1 \\
d-1
\end{array}\right)+e_{1}(I)\left(\begin{array}{c}
n+d-1 \\
d-1
\end{array}\right)+\text { lower terms, }
$$

which together with the observation in (5) proves the assertion.

In another application of this technique, we are going to derive a bound for the reduction number of an m-primary ideal of an arbitrary Noetherian local ring $(R, \mathfrak{m})$.

THEOREM 2.5. Let $(R, \mathfrak{m})$ be a Noetherian local of dimension $d$ and infinite residue field. For an $\mathrm{m}$-primary ideal $I$,

$$
r(I) \leqslant \lambda(R / J) \cdot d-2 d+1,
$$

where $J=\left(x_{1}, \ldots, x_{d}\right)$ is a minimal reduction of $I$.

LEMMA 2.6. Let $(R, \mathfrak{m})$ be a Noetherian local ring of dimension one. For any ideal I and for any parameter $x, v(I) \leqslant \lambda(R /(x))$.

Proof. We have used repeatedly the version of this result for Cohen-Macaulay rings. For the general case, set $L=H_{\mathfrak{m}}^{0}(R)$ and consider the two exact sequences

$$
\begin{aligned}
& 0 \rightarrow L \longrightarrow R \longrightarrow R^{\prime} \rightarrow 0, \\
& 0 \rightarrow L_{0}=I \cap L \longrightarrow I \longrightarrow I^{\prime} \rightarrow 0 .
\end{aligned}
$$


Tensoring by $R /(x)$, we get the exact sequences

$$
\begin{aligned}
& 0 \rightarrow{ }_{x} L \longrightarrow{ }_{x} R \longrightarrow{ }_{x} R^{\prime}=0 \longrightarrow L / x L \longrightarrow R / x R \rightarrow R^{\prime} / x R^{\prime} \rightarrow 0, \\
& 0 \rightarrow{ }_{x} L_{0} \longrightarrow{ }_{x} I \longrightarrow{ }_{x} I^{\prime}=0 \longrightarrow L_{0} / x L_{0} \longrightarrow I / x I \longrightarrow I^{\prime} / x I^{\prime} \rightarrow 0 .
\end{aligned}
$$

The second of the preceding sequences gives $v(I) \leqslant \lambda(I / x I)=\lambda\left(L_{0} / x L_{0}\right)+$ $\lambda\left(I^{\prime} / x I^{\prime}\right)$. Since $L$ is a module of finite length, $\lambda\left(L_{0} / x L_{0}\right)=\lambda\left({ }_{x} L_{0}\right) \leqslant \lambda\left({ }_{x} L\right)=$ $\lambda(L / x L)$, and therefore $v(I) \leqslant \lambda\left({ }_{x} L\right)+\lambda\left(I^{\prime} / x I^{\prime}\right)$. On the other hand, from the first sequence we have $\lambda(R / x R)=\lambda(L / x L)+\lambda\left(R^{\prime} / x R^{\prime}\right)$. Finally, $\lambda\left(I^{\prime} / x I^{\prime}\right) \leqslant \lambda\left(R^{\prime} / x R^{\prime}\right)$, since these are the multiplicities of the Cohen-Macaulay ring $R^{\prime}$ and its CohenMacaulay submodule $I^{\prime}$ (see Bruns and Herzog, 1993, § 4.7). Combining the inequalities we have the desired relation.

Proof of Theorem 2.5. As in the proof of Proposition 2.1, we set $J_{0}=$ $\left(x_{1}, \ldots, x_{d-1}\right)$ and estimate the number of generators of the ideal $M=I^{n} / J_{0}^{n}$ of the one-dimensional local ring $R / J_{0}^{n}$.

We use induction in $n$. Let $M_{0}$ be $M_{0}=M \cap J_{0}^{n-1} / J_{0}^{n}$. It will be enough to estimate the number of generators of factors such as $M^{\prime}=M / M_{0} \hookrightarrow J_{0}^{n-1} / J_{0}^{n}$ and add up. Note that the module $J^{n-1} / J_{0}^{n}$ is a homomorphic image of the module $F_{n-1}$ of $(n-1)$-forms in $d-1$ variables over the ring $R / J_{0}$. Therefore $M^{\prime}$ is a homomorphic image of a submodule of $F_{n-1}$, all of which can be generated by $\lambda(R / J) \cdot \operatorname{rank}\left(F_{n-1}\right)$ elements. The rest of the proof is as in Proposition 2.1.

If $R$ is Cohen-Macaulay, $\lambda(R / J)=e(I)$, the multiplicity of the ideal $I$. Observe in this case that $x$ does not have to be chosen in $I$, it could be a parameter for the maximal ideal of $R / J_{0}$. In particular, if $I \subset \mathfrak{m}^{s}$, we could replace in the formula $e(I)$ by $(e(I)) / s$.

\section{Number of Generators of Cohen-Macaulay Ideals}

Let $(R, \mathfrak{m})$ be a Cohen-Macaulay of dimension $d$ and let $I$ be an ideal of height $g>0$. If $I$ is a Cohen-Macaulay ideal, there are at least two approaches that can be used to bound the minimal number of generators of $I$ in terms of the multiplicity data.

A very general approach uses the method of extended multiplicities. These are numerical functions (denoted by $\operatorname{Deg}(\cdot)$ ) on modules that coincide with the classical multiplicity of local rings or graded modules (denoted by $\operatorname{deg}(\cdot)$ ). A typical expression from Doering et al. (1998) is

$$
v(I) \leqslant \operatorname{deg}(R)+(g-1) \operatorname{deg}(R / I)+\underbrace{(d-r-1)(\operatorname{Deg}(R / I)-\operatorname{deg}(R / I))}_{\text {non-Cohen-Macaulay correction }}
$$

where $\operatorname{Deg}(\cdot)$ is any extended degree function and $r=\operatorname{depth} R / I$. If $I$ is a Cohen-Macaulay ideal, $\operatorname{Deg}(R / I)=\operatorname{deg}(R / I)$, and we have (see also Valla (1981))

$$
v(I) \leqslant \operatorname{deg}(R)+(g-1) \operatorname{deg}(R / I) .
$$


The other method, which we already outlined, has a different character: Let $J_{0}$ be an ideal generated by a regular sequence of $g-1$ elements, $J_{0} \subset I$. Preferably $J_{0}$ should be part of a minimal reduction of $I$. We have

$$
v(I) \leqslant v\left(I / J_{0}\right)+v\left(J_{0}\right) \leqslant \operatorname{deg}\left(R / J_{0}\right)+g-1,
$$

since $I / J_{0}$ is a maximal Cohen-Macaulay module of rank 1 over the Cohen-Macaulay ring $R / J_{0}$. The issue is to relate $\operatorname{deg}\left(R / J_{0}\right)$ to $\operatorname{deg}(R / I)$.

Let us consider the case of an ideal $I$ that is equimultiple. The first approach is somewhat not sensitive to this additional information. Let us consider two applications of the second method. First, let $R$ be a Gorenstein ring and suppose $J=$ $\left(J_{0}, x\right)$ is a minimal reduction of $I$. Since $x$ is regular modulo $J_{0}$, we have $\operatorname{deg}\left(R / J_{0}\right) \leqslant \operatorname{deg}(R / J)$,

$$
v(I) \leqslant \operatorname{deg}(R / J)+g-1 .
$$

Consider the exact sequence

$$
0 \rightarrow(J: I) / J \longrightarrow R / J \longrightarrow R /(J: I) \rightarrow 0,
$$

where $(J: I) / J$ is the canonical module of $R / I$ and therefore $\operatorname{deg}((J: I) / J)=\operatorname{deg}$ $(R / I)$ that yields

$$
\operatorname{deg}(R / J)=\operatorname{deg}(R / I)+\operatorname{deg}(R /(J: I)) .
$$

If, for instance, $I$ is equimultiple of reduction number 1 , that is $I^{2}=J I, I \subset J: I$ and therefore $\operatorname{deg}(R / I) \geqslant \operatorname{deg}(R /(J: I))$, since they are both modules of dimension $d-g$. We thus obtain the estimate

$$
v(I) \leqslant 2 \operatorname{deg}(R / I)+g-1
$$

which is limited to this class of ideals.

The difference between the two approaches is more pronounced when we seek to estimate the multiplicity of a Cohen-Macaulay ideal of the form $R / I^{n}$ for some positive integer $n$. The point is that the multiplicity of $R / I^{n}$, for $n$ large, is a polynomial of degree $g$. On the other hand, using the second method we obtain

$$
\operatorname{deg}\left(R / J_{0}^{n}\right)=\operatorname{deg}\left(R / J_{0}\right)\left(\begin{array}{c}
n+g-1 \\
g-1
\end{array}\right),
$$

and therefore

$$
v\left(I^{n}\right) \leqslant \operatorname{deg}\left(R / J_{0}^{n}\right)+v\left(J_{0}^{n}\right) \leqslant \operatorname{deg}\left(R / J_{0}\right)\left(\begin{array}{c}
n+g-1 \\
g-1
\end{array}\right)+\left(\begin{array}{c}
n+g-2 \\
g-2
\end{array}\right),
$$

which in the equimultiple case gives

$$
v\left(I^{n}\right) \leqslant g ! \operatorname{deg}(R / \bar{I}) \operatorname{deg}(R)\left(\begin{array}{c}
n+g-1 \\
g-1
\end{array}\right)+\left(\begin{array}{c}
n+g-2 \\
g-2
\end{array}\right) .
$$


THEOREM 3.1. Let $R$ be a Cohen-Macaulay local ring with infinite residue field and let $I$ be an ideal of height $g>0$. If I is normally Cohen-Macaulay then

$$
\mathrm{r}(I) \leqslant g \cdot g ! \operatorname{deg}(R / \bar{I}) \operatorname{deg}(R)-2 g+1 .
$$

The proof is similar to that in Doering et al. (1998) in the m-primary case.

If the ideal $I$ is not normally Cohen-Macaulay the term $\operatorname{Deg}(R / I)-\operatorname{deg}(R / I)$ in (8) must be taken into account. The simplest case is that of an ideal $I$ of dimension one. One may proceed as follows, using the function $\operatorname{Deg}(\cdot)=h \operatorname{deg}(\cdot)$ of Vasconcelos (1998) $\left(A=R / J_{0}^{n}, \omega_{A}\right.$ the canonical module of $\left.A\right)$ :

$$
v\left(I^{n}\right) \leqslant v\left(J_{0}^{n}\right)+\operatorname{hdeg}\left(I^{n} / J_{0}^{n}\right),
$$

where

$$
\operatorname{Deg}\left(I^{n} / J_{0}^{n}\right)=\operatorname{deg}\left(I^{n} / J_{0}^{n}\right)+\operatorname{Deg}\left(\operatorname{Ext}_{A}^{1}\left(I^{n} / J_{0}^{n}, \omega_{A}\right)\right)+\operatorname{Deg}\left(\operatorname{Ext}_{A}^{2}\left(I^{n} / J_{0}^{n}, \omega_{A}\right)\right) .
$$

The last term vanishes and $\operatorname{Ext}_{A}^{1}\left(I^{n} / J_{0}^{n}, \omega_{A}\right)$ has finite length and therefore we may write

$$
\operatorname{Deg}\left(I^{n} / J_{0}^{n}\right)=\operatorname{deg}\left(I^{n} / J_{0}^{n}\right)+\lambda\left(\operatorname{Ext}_{A}^{1}\left(I^{n} / J_{0}^{n}, \omega_{A}\right)\right) .
$$

Finally an easy calculation shows that $\operatorname{Ext}_{A}^{1}\left(I^{n} / J_{0}^{n}, \omega_{A}\right) \simeq \operatorname{Ext}_{A}^{2}\left(R / I^{n}, \omega_{A}\right)$, a module which by local duality has the same length as $H_{\mathfrak{m}}^{0}\left(R / I^{n}\right)$. To sum up, we have the estimate

$$
v\left(I^{n}\right) \leqslant \operatorname{deg}\left(R / J_{0}\right)\left(\begin{array}{c}
n+d-2 \\
d-2
\end{array}\right)+\left(\begin{array}{c}
n+d-3 \\
d-3
\end{array}\right)+\lambda\left(H_{\mathfrak{m}}^{0}\left(R / I^{n}\right)\right) .
$$

The last term in this expression, $\lambda\left(H_{\mathrm{m}}^{0}\left(R / I^{n}\right)\right)$, is not very predictable. We will now give the method of Boratynski et al. (1979) that in dimension two is more suitable to the purpose here.

THEOREM 3.2. Let $(R, \mathfrak{m})$ be a Cohen-Macaulay local ring of dimension two and let $I$ be an ideal. If $\{x, y\}$ is a minimal reduction of $\mathrm{m}$ then

$$
v(I) \leqslant \operatorname{deg}(R)+\lambda(I:(x, y) / I) .
$$

Proof. Let $\mathbb{K}$ be the Koszul complex associated to $\{x, y\}$. For any finitely generated $R$-module $M$, the Euler characteristic of the complex $\mathbb{K}(M)=\mathbb{K} \otimes M$ is the multiplicity $e(x, y ; M)$ of the module $M$ (see Bruns and Herzog (1993) Theorem 4.7.4),

$$
e(x, y ; M)=\lambda\left(H_{0}(\mathbb{K}(M))-\lambda\left(H_{1}(\mathbb{K}(M))+\lambda\left(H_{2}(\mathbb{K}(M)) .\right.\right.\right.
$$

Setting $M=I$, observing that $v(M)=\lambda(M / \mathfrak{m} M) \leqslant \lambda(M /(x, y) M)=\lambda\left(H_{0}(\mathbb{K}(M))\right.$, and noting that $H_{1}(\mathbb{K}(I))=H_{2}(\mathbb{K}(R / I))=I:(x, y) / I$ and $H_{2}(\mathbb{K}(I))=0$, we obtain

$$
e(x, y ; I) \geqslant v(I)-\lambda(I:(x, y) / I) .
$$


From the additivity of the function $e(x, y ; \cdot)$, we have $e(x, y ; I) \leqslant \operatorname{deg}(R)$, giving rise to the desired inequality.

We may refine the bound (12) as follows:

COROLLARY 3.3. Let $(R, \mathfrak{m})$ be a Cohen-Macaulay local ring of dimension at least two and let $I$ be an ideal of dimension one. Let $J_{0}$ be a subideal of I generated by a regular sequence of $d-2$ elements. If $(x, y)$ is a minimal reduction of $\mathfrak{m}$ modulo $J_{0}$, then for all integers $n \geqslant 1$

$$
v\left(I^{n}\right) \leqslant \operatorname{deg}\left(R / J_{0}\right)\left(\begin{array}{c}
n+d-2 \\
d-2
\end{array}\right)+\left(\begin{array}{c}
n+d-3 \\
d-3
\end{array}\right)+\lambda\left(I^{n}:(x, y) / I^{n}\right) .
$$

\subsection{EQUIMULTIPLE IDEALS OF DIMENSION ONE}

Let $I$ be an ideal of dimension one as above but assume that $I$ is equimultiple. Let $J=\left(a_{1}, \ldots, a_{d-1}\right)$ be a minimal reduction. Let us discuss a reduction to the case when the ideal can be generated by $d$ elements. Consider the sequence

$$
0 \rightarrow I^{n} / J^{n} \hookrightarrow R / J^{n},
$$

that gives

$$
v\left(I^{n}\right) \leqslant v\left(I^{n} / J^{n}\right)+v\left(J^{n}\right) \leqslant \operatorname{deg}\left(R / J^{n}\right)+v\left(J^{n}\right),
$$

since $R / J^{n}$ is Cohen-Macaulay. As we have by Proposition 2.3 that

$$
\begin{aligned}
& \operatorname{deg}(R / J) \leqslant(d-1) ! \operatorname{deg}(R / \bar{I}) \operatorname{deg}(R), \\
& \operatorname{deg}\left(R / J^{n}\right) \leqslant(d-1) ! \operatorname{deg}(R / \bar{I}) \operatorname{deg}(R)\left(\begin{array}{c}
n+d-2 \\
d-1
\end{array}\right),
\end{aligned}
$$

we solve

$$
(d-1) ! \operatorname{deg}(R / \bar{I}) \operatorname{deg}(R)\left(\begin{array}{c}
n+d-2 \\
d-1
\end{array}\right)+\left(\begin{array}{c}
n+d-2 \\
d-2
\end{array}\right)<\left(\begin{array}{c}
n+d \\
d
\end{array}\right),
$$

and get a reduction $L$ generated by $d$ elements satisfying

$$
r=\mathrm{r}_{L}(I) \leqslant d ! \cdot \operatorname{deg}(R / \bar{I}) \operatorname{deg}(R)-2 d+1 .
$$

What is needed now is an estimate for the reduction number of $L$, since if $K$ is a minimal reduction of $L$, with $\mathrm{r}_{K}(L)=s$, it would follows that $r_{K}(I) \leqslant r+s$.

\section{Rees Algebra of the Conormal Module and Multiplicities}

Let $(R, \mathfrak{m})$ be a quasi-unmixed local ring of dimension $d$ and let $I$ be an ideal of codimension $g$ that is generically a complete intersection. We denote by $\mathcal{G}$ the associated graded ring of $I$. The conormal module $I / I^{2}$ of $I$ has rank $g$ and its Rees 
algebra $\mathcal{R}\left(I / I^{2}\right)$ is isomorphic to $\mathcal{G}$ modulo its $R / I$-torsion. For simplicity we denote it by $\mathcal{G}^{\prime}$,

$$
0 \rightarrow H(I) \longrightarrow \mathcal{G} \longrightarrow \mathcal{G}^{\prime} \rightarrow 0 .
$$

When $I$ is an ideal of dimension 1 , or $R / I$ has isolated singularities, $H(I)=H_{\mathrm{m}}^{0}(\mathcal{G})$. In this case, from the exact sequence in which the associated primes of $\mathcal{G}^{\prime}$ do not contain those of $H(I)$, we obtain the equality of arithmetic degrees

$$
\operatorname{arith}-\operatorname{deg}(G)=\operatorname{arith}-\operatorname{deg}(H(I))+\operatorname{arith}-\operatorname{deg}\left(\mathcal{G}^{\prime}\right) .
$$

Throughout this section, in order to have this description of $H(I)$ we shall assume that $I / I^{2}$ is torsionfree on the punctured spectrum. We want to make the case that the examination of both $H(I)$ and of $\mathcal{G}^{\prime}$ play a role in the determination of the reduction number of $I$ in terms of the arithmetic degree of $\mathcal{G}$. A first observation is that if $J$ is a minimal reduction of $I$, then $H(I)$ and $\mathcal{G}^{\prime}$ are graded $R[J t]$-modules and we will seek the degrees of the equations of integral dependence of the elements of $R[I t]$ acting as endomorphisms of these modules.

We will now pay attention to some of the general properties of $H(I)$ of $\mathcal{G}^{\prime}$. We note that $\operatorname{dim} \mathcal{G}^{\prime}=\operatorname{dim} R=d$, and that

$$
\operatorname{dim} \mathcal{G}^{\prime} / \mathfrak{m} \mathcal{G}^{\prime}<d,
$$

since $\mathfrak{m}$ contains regular elements on $\mathcal{G}^{\prime}$. In the theory of Rees algebras of modules, the dimension of the special fiber of $\mathcal{G}^{\prime}$ is called the analytic spread of the module

$$
\ell\left(I / I^{2}\right)=\operatorname{dim} \mathcal{G}^{\prime} / \mathfrak{m} \mathcal{G}^{\prime} .
$$

DEFINITION 4.1. The inertial analytic spread of $I$ is the dimension of the module $H(I)$. We will denote it by $h(I)=\operatorname{dim} H(I)$.

A first observation is that since $H(I)$ is annihilated by a power of $\mathfrak{m}$, it admits a filtration whose factors are modules over $\mathcal{G} \otimes R / \mathfrak{m}=\mathcal{F}(I)$. It follows that

$$
h(I)=\operatorname{dim} H(I) \leqslant \operatorname{dim} \mathcal{F}(I)=\ell(I) .
$$

A more concrete elementary observation is the following:

PROPOSITION 4.2. Let $(R, \mathfrak{m})$ be a Noetherian local ring of dimension $d$ and let $I$ be an ideal. If $\ell(I)=d$, then $h(I)=\ell(I)$.

Proof. Using the Artin-Rees lemma, choose $s$ to be large enough so that $H \cap \mathfrak{m}^{s} \mathcal{G}=0$. This leads to the exact sequence

$$
0 \rightarrow H \longrightarrow \mathcal{G} / \mathfrak{m}^{s} \mathcal{G} \longrightarrow \mathcal{G}^{\prime} / \mathfrak{m}^{s} \mathcal{G}^{\prime} \rightarrow 0
$$


which shows that

$\operatorname{dim} H \leqslant \operatorname{dim} \mathcal{G} / \mathfrak{m}^{s} \mathcal{G}=\max \left\{\operatorname{dim} H, \operatorname{dim} \mathcal{G}^{\prime} / \mathfrak{m}^{s} \mathcal{G}^{\prime}\right\}$.

But $\operatorname{dim} \mathcal{G} / \mathfrak{m}^{s} \mathcal{G}=\operatorname{dim} \mathcal{G} / \mathfrak{m} \mathcal{G}$, and $\operatorname{dim} \mathcal{G}^{\prime} / \mathfrak{m}^{s} \mathcal{G}^{\prime}<d$ since $\mathfrak{m}$ does not consist of zero divisors of $\mathcal{G}^{\prime}$. This shows that we must have $h(I)=\ell(I)$, as desired.

COROLLARY 4.3. Let I be a non-m-primary ideal such that all the associated primes of $\mathcal{G}$ have dimension $d$ and $H(I) \neq 0$. Then $h(I)=\ell(I)=d$ and $\operatorname{dim} \mathcal{G}^{\prime}=d$. In particular $\operatorname{deg}(\mathcal{G})=\operatorname{deg}(H)+\operatorname{deg}\left(\mathcal{G}^{\prime}\right)$.

Proof. If $H(I) \neq 0$, it is a module of the same dimension as $\mathcal{G}$, since the latter is equidimensional. From Proposition 4.2, we have that $d=h(I) \leqslant \ell(I)$, which proves the first assertion.

Suppose now that $\operatorname{dim} \mathcal{G}^{\prime}<d$. This means that $H(I)$ is an ideal of height at least 1 and therefore must contain regular elements of $\mathcal{G}$. On the other hand, $\mathfrak{m}^{s} H=0$, which implies that $\mathfrak{m}^{s} \subset I$, contradicting the assumption that $I$ is not a primary ideal. The asserted addition formula follows from the ways one computes multiplicities.

PROPOSITION 4.4. Let $(R, \mathfrak{m})$ be a Cohen-Macaulay local ring of dimension d, let I be an unmixed ideal of codimension $g$ and $\ell(I)=d$ that is of linear type on the punctured spectrum of $R$. If $H(I) \neq 0$, then $\ell\left(I / I^{2}\right)=d-1$.

Proof. First, note that as $\ell(I)=d, \mathfrak{m G}$ has codimension 0 and it is not a nilpotent ideal. On the other hand, $H(I)$ is not a nilpotent ideal either as this would yield $\mathcal{G}_{\text {red }}=\mathcal{G}_{\text {red }}^{\prime}$ and therefore $\ell(I)$ would be at most $d-1$. On the other hand, since $R$ is Cohen-Macaulay, the ring $\mathcal{G}$ is connected in codimension one (see Brodmann, 1986, 2.5). This means that if $A, B$ are nonnilpotent ideals with $A \cdot B=0$, then height $(A+B) \leqslant 1$. Applying this to the equation $\mathfrak{m}^{s} H(I)=0$, we have that height $\left(\mathfrak{m}^{s} \mathcal{G}+H(I)\right) \leqslant 1$, and therefore $\operatorname{dim} \mathcal{G}^{\prime} / \mathfrak{m}^{s} G r^{\prime} \geqslant d-1$, as desired.

These observations will be useful for two reasons. First, we can use the theory of Cayley-Hamilton polynomials to bound the degrees of endomorphisms on $H(I)$; second, the fact that $\ell\left(I / I^{2}\right)$ is usually smaller than $\ell(I)$ permit us to get a simpler reduction for the module than the ideal $I$ itself allows.

\subsection{IDEALS OF DIMENSION ONE}

The first application of this setting is:

PROPOSITION 4.5. Let $(R, \mathfrak{m})$ be a Cohen-Macaulay local ring and let I be an ideal of dimension 1 that is generically a complete intersection. Let $H=H_{\mathfrak{m}}^{0}(\mathcal{G})$. If $H \neq 0$ then $\operatorname{dim} H=d$ and the algebra defined by

$$
0 \rightarrow H \longrightarrow \mathcal{G} \longrightarrow \mathcal{G}^{\prime} \rightarrow 0
$$


satisfies $\operatorname{dim} \mathcal{G}^{\prime}=d$ and $\operatorname{deg}(R / I)=\operatorname{deg}\left(\mathcal{G}^{\prime}\right)=\operatorname{deg}(\mathcal{G})-\operatorname{deg}(H)$.

Proof. We only have to show that $\operatorname{deg}(R / I)=\operatorname{deg}\left(\mathcal{G}^{\prime}\right)$. The $R / I$-module

$$
E=I / I^{2} \text { (modulo torsion) }
$$

admits a reduction $F \subset E$ which is a free module. Thus the Rees algebras $\mathcal{R}(F) \subset$ $\mathcal{R}(E)$ have the same multiplicity. Since $\mathcal{R}(F) \simeq R / I\left[T_{1}, \ldots, T_{d-1}\right]$, we get the asserted equality of multiplicities.

THEOREM 4.6. Let $(R, \mathfrak{m})$ be a Cohen-Macaulay local ring and let I be an ideal of dimension 1 that is generically a complete intersection. Suppose further that $R$ contains a field of characteristic zero. Then

$$
\mathrm{r}(I)<\operatorname{deg}(\mathcal{G}) .
$$

Proof. We may assume that $R$ is a complete local ring. Let $J$ be a minimal reduction of $I$ and view the terms of the exact sequence

$$
0 \rightarrow H \longrightarrow \mathcal{G} \longrightarrow \mathcal{G}^{\prime} \rightarrow 0
$$

as modules over the Rees algebra $R[J t]$. We can also view $H$ as a module over a ring of polynomials $k\left[T_{1}, \ldots, T_{d}\right]$ which is actually the special fiber of $R[J t]$. This happens because $H$ is a module annihilated by a power of the maximal ideal $\mathfrak{m}^{r}$ and therefore is defined over the Artinian ring $R / \mathrm{m}^{r}$. Since $R / \mathfrak{m}$ has characteristic zero, we can find a field of representatives of $R / \mathrm{m}^{r}$.

After noting that the image of $J t$ in $\mathcal{G}^{\prime}$ defines a reduction of the module $E$ above, we can choose a minimal reduction $F$ of $E$ contained in $J t \mathcal{G}^{\prime}$. As $R$ contains a field $k$ of representatives, there is a parameter $z$ such that the inclusion $k[[z]] \subset R / I$ makes $R / I$ free over $k[[z]]$ of $\operatorname{rank} \operatorname{deg}\left(\mathcal{G}^{\prime}\right)=\operatorname{deg}(R / I)$. After changing notation in the original $T_{i}$, let $T_{1}, \ldots, T_{d-1}$ be a set of generators of the module $F$. The ring $\mathcal{G}^{\prime}$ is a finitely generated torsionfree module over $A=k[[z]]\left[T_{1}, \ldots, T_{d-1}\right]$, of rank $\operatorname{deg}(R / I)$.

Finally let $a t$ be an element of $I t$ and consider the mapping it induces as an endomorphism of $H$ and of $\mathcal{G}^{\prime}$. Applying the techniques of Cayley-Hamilton theorems of Vasconcelos (1998b, Section 9.3), we get polynomials $f(T)$ and $g(T)$ monic and graded over $R[J t]$, of degrees $\operatorname{deg}(H)$ and $\operatorname{deg}\left(\mathcal{G}^{\prime}\right)$ respectively, such that $f(a t) H=0$ and $g(a t) \mathcal{G}^{\prime}=0$. Thus $f(a t) g(a t) \mathcal{G}=0$, which shows that $p(a t)=f(a t) g(a t)$ is the null element of $\mathcal{G}$. By lifting to $\mathcal{R}$ this gives an equation of integral dependence of $a$ with respect to $J$ of degree $n=\operatorname{deg}(H)+\operatorname{deg}\left(\mathcal{G}^{\prime}\right)$,

$$
a^{n}+b_{1} a^{n-1}+\cdots+b_{n} \in(I R[I t])_{n}=I^{n+1}, \quad b_{i} \in J^{i} .
$$

In characteristic zero this implies, as remarked earlier, the desired inequality $\mathrm{r}(I)<\operatorname{deg}(\mathcal{G})$. 


\subsection{GORENSTEIN IDEALS OF DIMENSION TWO}

It becomes considerably more complicated when the ideal has dimension greater than one. To simplify we assume that $R$ is a Gorenstein local $\operatorname{ring}, \operatorname{dim} R / I=2$, that $I$ is a perfect Gorenstein ideal and $R / I$ is normal.

Let us make some general observations first. Let $J$ be a minimal reduction of $I$. If $\ell(I)<d, I$ is a complete intersection. Indeed, if $J$ is generated by $d-1$ elements by Vasconcelos (1994, Corollary 5.3.5), its associated primes have codimension at most $d-1$, which is impossible since $R / I$ is normal.

We may then assume that $I$ has a minimal reduction $J=\left(a_{1}, \ldots, a_{d}\right)$. In particular this implies that $H_{\mathrm{m}}^{0}(\mathcal{G}) \neq 0$. The module

$$
E=I / I^{2} /(\text { modulo torsion) }
$$

has analytic spread $d-1$, so that $d-1$ elements of $J t$ map into a reduction $F$ of $E$. Indeed a minimal reduction of $E$ cannot be a free module $F$ since then $\mathcal{G}^{\prime}$ would be integral over a ring of polynomials over a normal ring. This would imply that $E$ is also free which in turn would mean that

$$
I / I^{2} \simeq(R / I)^{d-2} \oplus L, \quad L \neq 0,
$$

a condition that would say that $I$ contains a regular sequence of $d-1$ elements. Therefore we have $\ell(E)=d-1$.

We claim that $\mathcal{G}^{\prime}$ has a reduction that is a hypersurface ring over $R / I$,

$$
\mathcal{R}(F)=R / I\left[T_{1}, \ldots, T_{d-1}\right] /(f), \quad f=\sum_{i=1}^{d-1} a_{i} T_{i} .
$$

Let $F$ be a minimal reduction of $E$. $F$ is a torsionfree module with a presentation

$$
0 \rightarrow K \longrightarrow(R / I)^{d-1} \longrightarrow F \rightarrow 0,
$$

where $K$ is a reflexive module of rank 1. Let $f \in(R / I)^{d-1}$ be a nonzero element of $K$, and let $L$ be the ideal generated by its coordinates as an element of $(R / I)^{d-1}$. Note that $L^{-1} f \subset K$. Checking at the localizations of $R / I$ at height one primes, it follows easily that these modules are the same.

We now make use of the fact that $E$ is an orientable module, and therefore so will be its reductions. From the exact sequence above, $L^{-1} f$ is an orientable module as well. Since it is reflexive, of rank one, it must be principal.

\subsection{MULTIPLICITY OF ALMOST COMPLETE INTERSECTIONS}

Let $(R, \mathfrak{m})$ be a Cohen-Macaulay local ring and let $E$ be a torsionfree $R$-module of rank $r>0$ with $\ell(E)=r+1$. We assume that $E$ is an orientable module. This will imply that any minimal reduction $F$ has a free presentation

$$
0 \rightarrow R \longrightarrow R^{r+1} \longrightarrow F \rightarrow 0 .
$$


We will assume further that the Rees algebra of $F$ is an almost complete intersection, which means that

$$
\mathcal{R}(F)=R\left[T_{1}, \ldots, T_{r+1}\right] /(f), \quad f=a_{1} T_{1}+\cdots+a_{r+1} T_{r+1}
$$

where $\left(a_{1}, \ldots, a_{r+1}\right)$ is an ideal of grade at least 2 . From this description one has

$$
\operatorname{deg}(\mathcal{R}(E))=\operatorname{deg}(\mathcal{R}(F))=\operatorname{deg} R\left(T_{1}, \ldots, T_{r+1}\right) /(f) .
$$

We want however a different expression for this multiplicity. Let us explain the goal of how to obtain a bound for the reduction number of $E$ in terms of this multiplicity. We do this using a number of elementary observations.

Since the ring $R$ is Cohen-Macaulay and $\mathcal{R}(F)$ is Cohen-Macaulay and the ideal generated by its 1 -forms has height $r$, there is (we are assuming that the residue field of $R$ is infinite) a submodule $\tilde{F}$ of $F$ generating a regular sequence of height $r$. Furthermore its generators can be chosen so that $\operatorname{deg}(\mathcal{R}(F))=\operatorname{deg}(\mathcal{R}(F) /(\tilde{F}))$.

PROPOSITION 4.7. The R-module $C_{n}$ defined by the exact sequence

$$
0 \rightarrow \tilde{F}^{n} \longrightarrow F^{n} \longrightarrow C_{n} \rightarrow 0,
$$

has multiplicity

$$
\operatorname{deg}\left(C_{n}\right)=\left(\begin{array}{c}
n+r-1 \\
r
\end{array}\right) \operatorname{deg}\left(C_{1}\right) .
$$

Proof. We note that the modules $F^{n}$ all have projective dimension 1 so that the projective dimension of $C_{n}$ is also 1 . So for the purpose of putting together $\operatorname{deg}\left(C_{n}\right)$ through the usual associativity formula

$$
\operatorname{deg}\left(C_{n}\right)=\sum_{\text {height } \mathfrak{p}=1} \lambda\left(\left(C_{n}\right)_{\mathfrak{p}}\right) \operatorname{deg}(R / \mathfrak{p}),
$$

we may assume that $\operatorname{dim} R=1$.

We recall that we may reduce to the case of modules over discrete valuation rings, and the assertion is a simple calculation of determinants.

PROPOSITION 4.8. Suppose further that $\operatorname{dim} R=2$ and set $a=\operatorname{deg}\left(C_{1}\right)$. Then

$$
r(E) \leqslant(r+1) a-2(r+1)-1 .
$$

Proof. We note that the module $E^{n} / \tilde{F}^{n}$ is Cohen-Macaulay of dimension 1. Its multiplicity is the same as that of $F^{n} / \tilde{F}^{n}$. This leads to the inequalities

$$
\begin{aligned}
v\left(E^{n}\right) & \leqslant v\left(E^{n} / \tilde{F}^{n}\right)+v\left(\tilde{F}^{n}\right) \\
& \leqslant \operatorname{deg}\left(E^{n} / \tilde{F}^{n}\right)+v\left(\tilde{F}^{n}\right) \\
& \leqslant a\left(\begin{array}{c}
n+r-1 \\
r
\end{array}\right)+\left(\begin{array}{c}
n+r-1 \\
r-1
\end{array}\right) .
\end{aligned}
$$


To apply Eakin and Sathaye (1976) again (we may have to replace $F$ by another minimal reduction $\left.F^{\prime}\right)$, since $\ell(F)=r+1$, is will suffice to set $n=\mathrm{r}(E)+1$ such that

$$
\left(\begin{array}{c}
n+r+1 \\
r+1
\end{array}\right)>a\left(\begin{array}{c}
n+r-1 \\
r
\end{array}\right)+\left(\begin{array}{c}
n+r-1 \\
r-1
\end{array}\right)
$$

This is equivalent to

$$
n>(r+1) a-2(r+1),
$$

which is the desired bound for the reduction number of $E$.

We must now relate $a=\operatorname{deg}\left(C_{1}\right)$ to the multiplicity of $\mathcal{R}(F)$. We use the additivity formula on $\mathcal{R}(F) /(\tilde{F})$. Let $\mathfrak{B}$ be a minimal prime of $(\tilde{F}), \mathfrak{p}=R \cap \mathfrak{B}$. Note that $\mathfrak{p} \neq \mathfrak{m}$ since $\tilde{F}$ is generated by $r$ elements in a minimal generating set for $F$. In particular $C_{1}$ is a cyclic module.

There will be two kinds of minimal primes $\mathfrak{P}$ of $(\tilde{F})$, depending on whether $\mathfrak{p}=R \cap \mathfrak{P}$ has height 0 or 1 . If $\mathfrak{p}$ is a minimal prime of $R, \mathfrak{B}=(\mathfrak{p}, F)$. In the other case, $\mathfrak{P}=(\mathfrak{p}, \tilde{F})$, where $\mathfrak{p}$ is an associated prime of $C_{1}$.

We can now put together the multiplicity formula:

$$
\begin{aligned}
\operatorname{deg}(\mathcal{R}(F) /(\tilde{F}))= & \sum_{\text {height } \mathfrak{p}=0} \lambda\left((\mathcal{R}(F) / \tilde{F})_{\mathfrak{R}}\right) \operatorname{deg}(\mathcal{R}(F) / \mathfrak{R})+ \\
& +\sum_{\text {height } \mathfrak{p}=1} \lambda\left((\mathcal{R}(F) / \tilde{F})_{\mathfrak{K}}\right) \operatorname{deg}(\mathcal{R}(F) / \mathfrak{R})
\end{aligned}
$$

The first partial summation, since $\mathfrak{P}=(\mathfrak{p}, F)$, gives

$$
\sum_{\text {height } \mathfrak{p}=0} \lambda\left((\mathcal{R}(F) /(F))_{\mathfrak{p}}=R_{\mathfrak{p}}\right) \operatorname{deg}(R / \mathfrak{p})=\operatorname{deg}(R / I) .
$$

In the other partial summation, one has

$$
\lambda\left((\mathcal{R}(F) /(F))_{\mathfrak{s}}\right)=\lambda\left(\left(C_{1}\right)_{\mathfrak{p}}\right),
$$

and the degree factor is at least 1 . Altogether we obtained:

PROPOSITION 4.9. The following estimation holds

$$
\operatorname{deg}(\mathcal{R}(F))=\operatorname{deg}(\mathcal{R}(F) /(\tilde{F})) \geqslant \operatorname{deg}(R / I)+a .
$$

As a consequence, we derive the following bound for the reduction number of the Gorenstein ideal that we treated:

THEOREM 4.10. Let $R$ be a Gorenstein local ring of dimension $d$ and let $I$ be a perfect Gorenstein ideal of dimension two such that $R / I$ is normal. Suppose in addition 
that the residue field of $R$ has characteristic zero. The following bound for the reduction number of I holds

$$
r(I) \leqslant(d-1) \cdot \operatorname{deg}(\mathcal{G})-4 d+5 .
$$

Proof. We are going to bound the reduction number of $I$ by gluing it from the reduction number of $E$ and the multiplicity of $H(I)$ in the exact sequence

$$
0 \rightarrow H(I) \longrightarrow \mathcal{G} \longrightarrow \mathcal{G}^{\prime} \rightarrow 0 .
$$

For each element $z \in \mathcal{G}$, we have a monic, homogeneous, polynomial $f(z)$ with coefficients in a minimal reduction of $I$ (in fact, any minimal reduction) such that $f(z) H(I)=0$. We proved earlier that $f$ can be chosen of degree at most $e(H(I)) \leqslant \operatorname{deg}(\mathcal{G})$.

On the other hand, we also proved that $E^{s+1}=F E^{s}$, where (recall $r=d-2$ )

$$
s=(d-1) a-2(d-1)+1 .
$$

Lift $F$ to a minimal reduction $J \subset I$. This is feasible with the assumption that $R$ has an infinite residue field and the manner in which the reductions $F$ (or $F^{\prime}$ ) and $J$ are chosen as generic linear combinations of a set of generators of $I$. Thus for any element $Z \in \mathcal{G}_{1}$, we have that $z=Z^{s+1}-g(Z) \in H(I)$, with the class of $g(Z)$ in $\mathcal{R}(E)$ lying in $F E^{s}$. Now we find an equation for $z$ as described earlier. Altogether we have an equation of integral dependence for $Z$ over $J t \mathcal{G}$, of degree $\leqslant s+1+e(H(I))$.

Since we proved that $a \leqslant \operatorname{deg}(\mathcal{R}(F))=\operatorname{deg}(\mathcal{G})-\operatorname{deg}(H(I))$, we obtain a reduction number

$$
\begin{aligned}
\mathrm{r}(I) & \leqslant \operatorname{arith}-\operatorname{deg}(H(I))+(d-1) a-2(d-1)+1 \\
& \leqslant \operatorname{arith}-\operatorname{deg}(H(I))+(d-1)(\operatorname{deg}(\mathcal{G})-\operatorname{arith}-\operatorname{deg}(H(I))-2 d+3 \\
& \leqslant+(d-1) \operatorname{deg}(\mathcal{G})-(d-2) \operatorname{arith}-\operatorname{deg}(H(I))-2 d+3 \\
& \leqslant(d-1) \cdot \operatorname{deg}(\mathcal{G})-4 d+5 .
\end{aligned}
$$

This is very similar to the formula of Doering et al. (1998) that considers the primary case.

\section{Acknowledgement}

The author was partially supported by a grant from the National Science Foundation.

\section{References}

Boratynski, M., Eisenbud, D. and Rees, D. (1979) On the number of generators of ideals in local Cohen-Macaulay rings, J. Algebra 57, 77-81.

Brodmann, M. (1986) A few remarks on blowing-up and connectedness, J. reine angew. Math. 370, 52-60.

Bruns, W. and Herzog, J. (1993) Cohen-Macaulay Rings, Cambridge Univ. Press. 
Doering, L. R., Gunston, T. and Vasconcelos, W. V. (1998) Cohomological degrees and Hilbert functions of graded modules, Amer. J. Math. 120, 493-504.

Eakin, P. and Sathaye, A. (1976) Prestable ideals, J. Algebra 41, 439-454.

Johnston, B. and Katz, D. (1995) Castelnuovo regularity and graded rings associated to an ideal, Proc. Amer. Math. Soc. 123, 727-734.

Lech, C. (1960) Note on multiplicities of ideals, Arkiv Mat. 4, 63-86.

Rees, D. (1961) $\alpha$-transforms of local rings and a theorem on multiplicities of ideals, Math. Proc. Cambridge Philos. Soc. 57, 8-17.

Rossi, M. E., Valla, G. and Vasconcelos, W. V. (2001) Maximal Hilbert functions, Results in Math. 39, 99-114.

Valla, G. (1981) Generators of ideals and multiplicities, Comm. Algebra 9, 1541-1549.

Vasconcelos, W. V. (1994) Arithmetic of Blowup Algebras, London Math. Soc. Lecture Note Ser. 195, Cambridge Univ. Press.

Vasconcelos, W. V. (1998a) Cohomological degrees of graded modules, In: Six Lectures on Commutative Algebra, Progr. in Math. 166, Birkhäuser, Boston, 1998, 345-392.

Vasconcelos, W. V. (1998b) Computational Methods in Commutative Algebra and Algebraic Geometry, Springer, Heidelberg.

Vasconcelos, W. V. (1999) The reduction numbers of an ideal, J. Algebra 216 652-664. 